\title{
VLPC SINGLE CASSETTE CRYOSTAT CHRISTMAS TREE TEMPERATURE AS RELATED TO ANNULUS FLOW AND LHE LEVEL
}

DANIEL OLIS

$4 / 23 / 93$

D-ZERO ENGINEERING NOTE 3823.112-EN-339

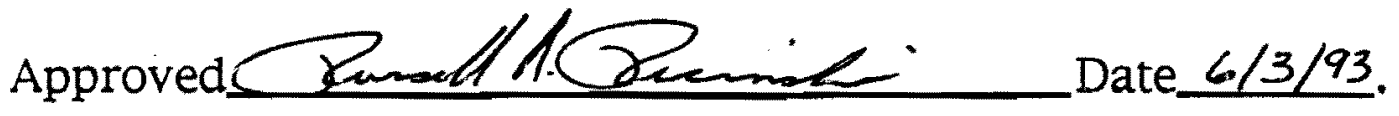




\section{VLPC SINGLE CASSETTE CRYOSTAT CHRISTMAS TREE TEMPERATURE AS RELATED TO ANNULUS FLOW AND LHE LEVEL Dan Olis $4 / 21 / 93$}

Data taken from tests of annulus shield flow versus Christmas tree temperature show that the temperature of the tree is controlled by the annulus flow and the LHe level in the reservoir. Graphs indicating this are shown in Figures 1 and 2. An equation determined from the data taken on $4 / 19$ to model the flow and LHe level dependence of tree temperature is as follows:

$$
\begin{aligned}
& \mathrm{T}=\mathrm{AL}+\mathrm{BF}+\mathrm{C} \\
& \mathrm{T}=\text { tree temperature }(\mathrm{K}) \\
& A=-0.0055(K / \%) \\
& \mathrm{L}=\mathrm{LHe} \text { Level }(\%) \\
& B=-1.166 \text { (K/scth air) } \\
& F=\text { annulus flow (scth air) } \\
& \mathrm{C}=7.889(\mathrm{~K}) \\
& 10 \%\left(0.6^{\prime \prime}\right)<L<65 \%\left(3.9^{\prime \prime}\right) \\
& 0.5<F<1.0 \mathrm{scfh}
\end{aligned}
$$

From the above equation it is evident that shield flow has a significant effect on tree temperature while the percent of $\mathrm{LHe}$ in the reservoir is much less significant. The following illustrates the temperature's relative sensitivity to the two variables:

$$
\begin{aligned}
\triangle \text { flow } & =0.5 \mathrm{scth} \text { gives } \triangle \mathrm{T}=0.58 \mathrm{~K} \\
\triangle \text { level } & =40 \% \text { LHe gives } \triangle \mathrm{T}=0.22 \mathrm{~K}
\end{aligned}
$$

A graph of Temperature Calculated vs. Temperature Measured in Figure 3 shows the degree to which the equation conforms to the data taken on $4 / 19$. This test data is included in the appendix. The measured temperature, calculated temperature, and the percent of error between the two is shown among the data.

Figure 3 and the 'Temp.\%Error' column in the data indicate the degree of the equation's accuracy. When determining the value of the above equation it is important to consider Figure 4. The graph shows Temperature vs. Annulus Flow data collected on a number of different days. Note that data collected from day to day have similar slopes yet different $y$ intercepts. This means that the degree to which flow effects temperature remained relatively constant from day to day, yet some uriknown variable in temperature control remains. Initially it seems possible that variations in cryostat pressure might be the third variable. But the maximum possible pressure change of 4 psig within the cryostat only accounts for a $0.24 \mathrm{~K}$ temperature difference. One other theory is that the $\mathrm{GHe}$ used to apply positve pressure to the cassette space is leaking out the cassette top and is causing the change in y-intercepts. A leak in the cassette top would allow warm GHe to enter the cassette volume. Furhter tests will be done to see if this cassette leak is in fact the problem.

The above equation cannot be applied at some instant to determine the annulus flow required at some LHe level to produce a desired tree temperature. Rather its value is that it shows the relative contribution of the two variables to the temperature and the temperature's sensitivity to them. It seems regulation of the tree temperature would best be achieved by providing a feedback loop between temperature and shield flow while maintaining a relatively steady $( \pm 5 \%) \mathrm{LHe}$ level. As one last note, I found that regulating the LHe level with the inlet valve caused disturbances in the cryostat that resulted in temperature drops as great as 0.2 $K$. To prevent this, when LHe levels fell too low, I would remove the boil-off hose from the regulator for a few minutes until the LHe level was back to a satisfactory level. From this it seems that the LHe level can be controlled by the boil-off regulator with less upset than by adjusting the LHe inlet valve. 


\section{Temp. \& LHe Level vs. Time}

annulus flow $=0.79 \mathrm{scth}$ air

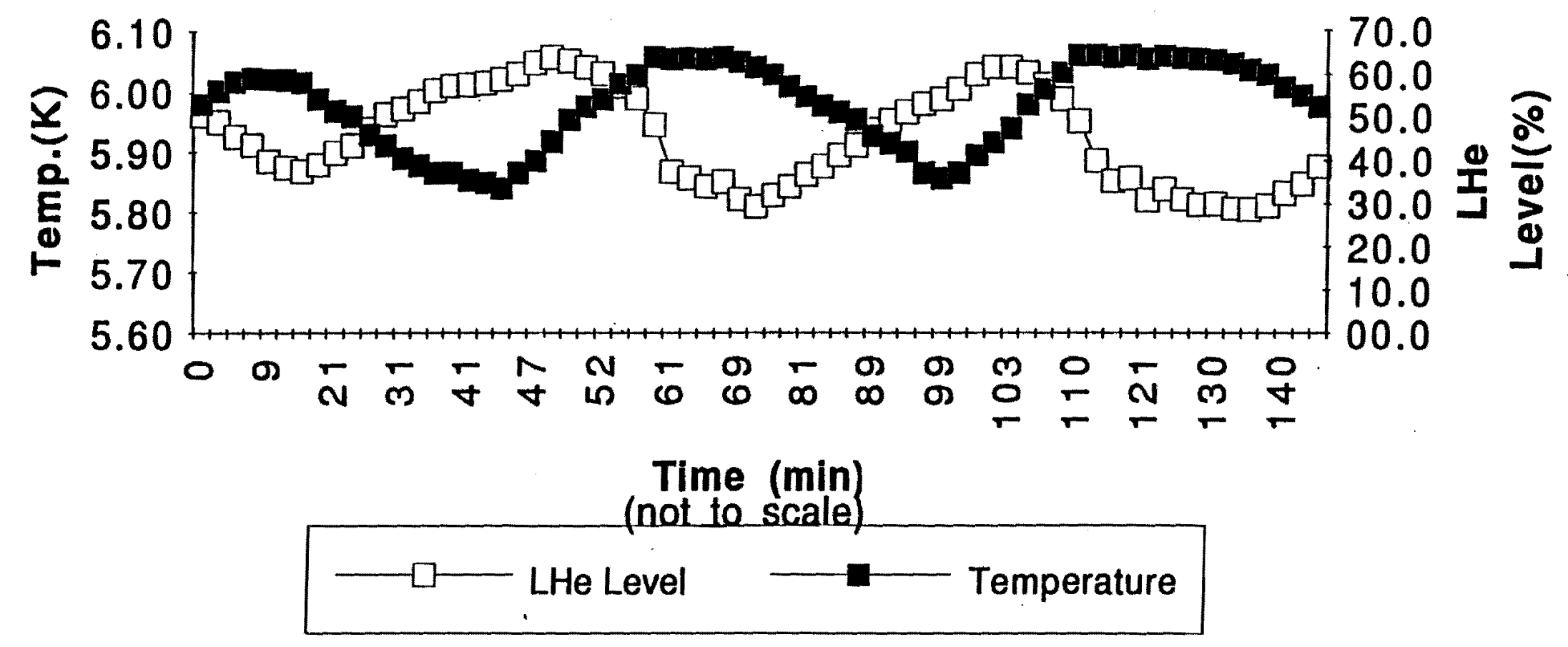




\section{Temp.\&LHeLevel v. Time}

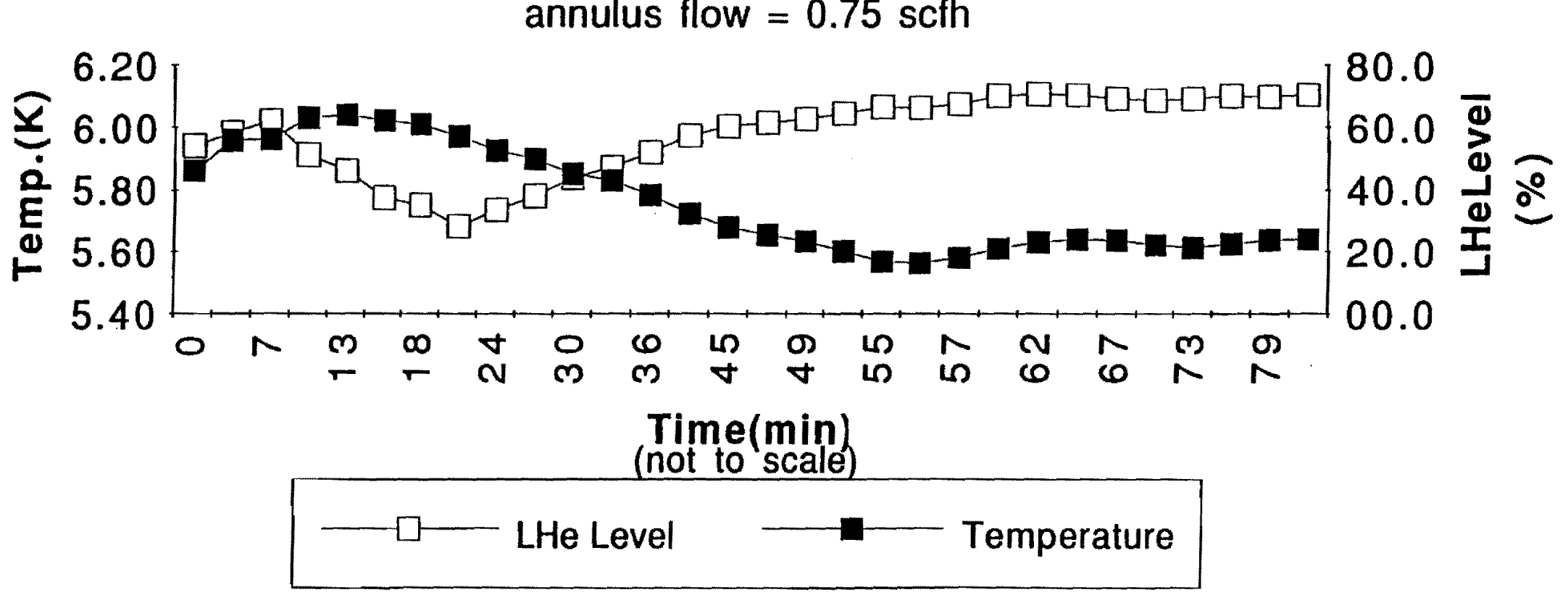


Temperature Calculated vs. Temperature Measured

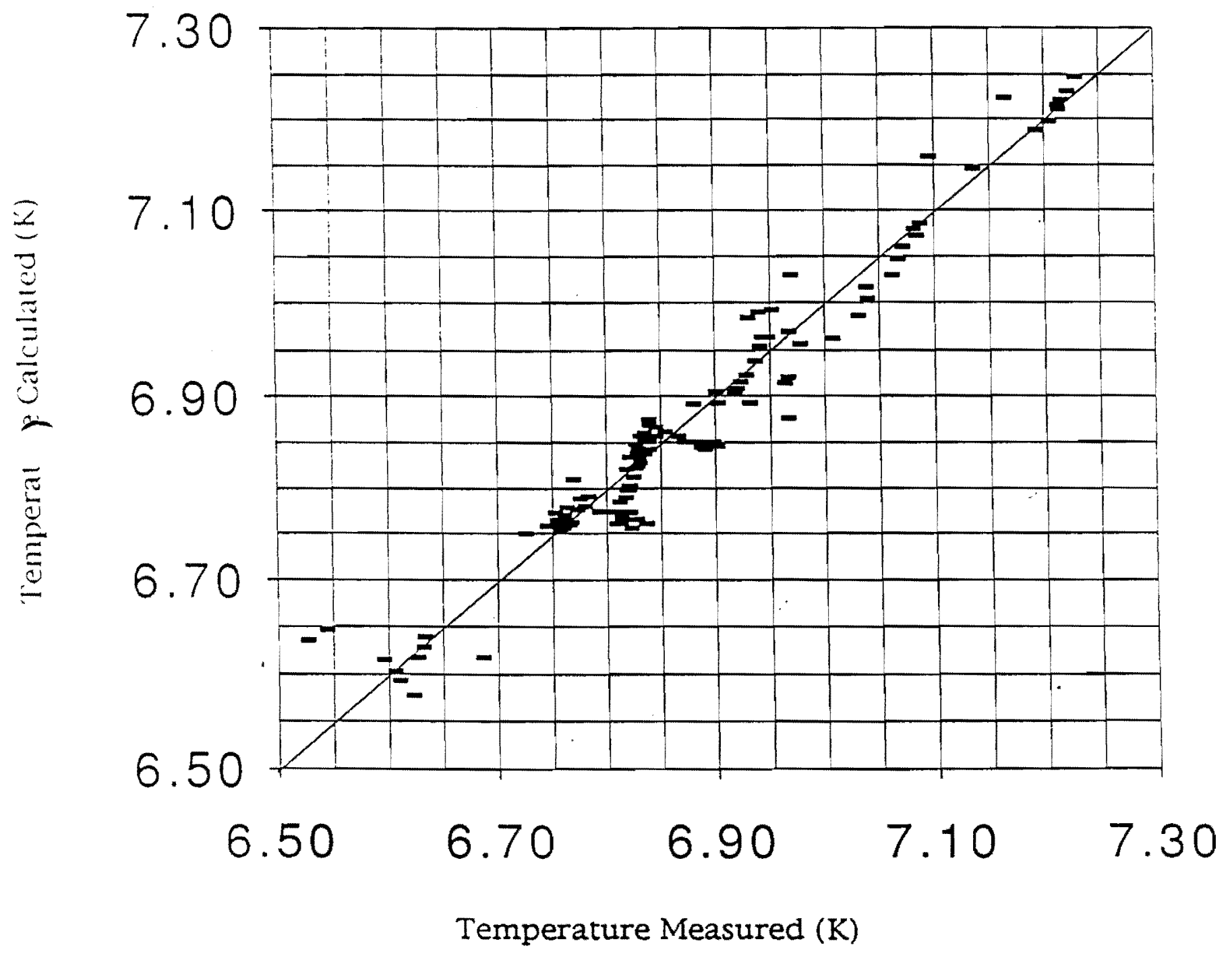

Note: Graph is of data collected on 4/19 and does not include test start-up data points.

Figure 3. 


\section{Temperature vs. Annulus Flow}

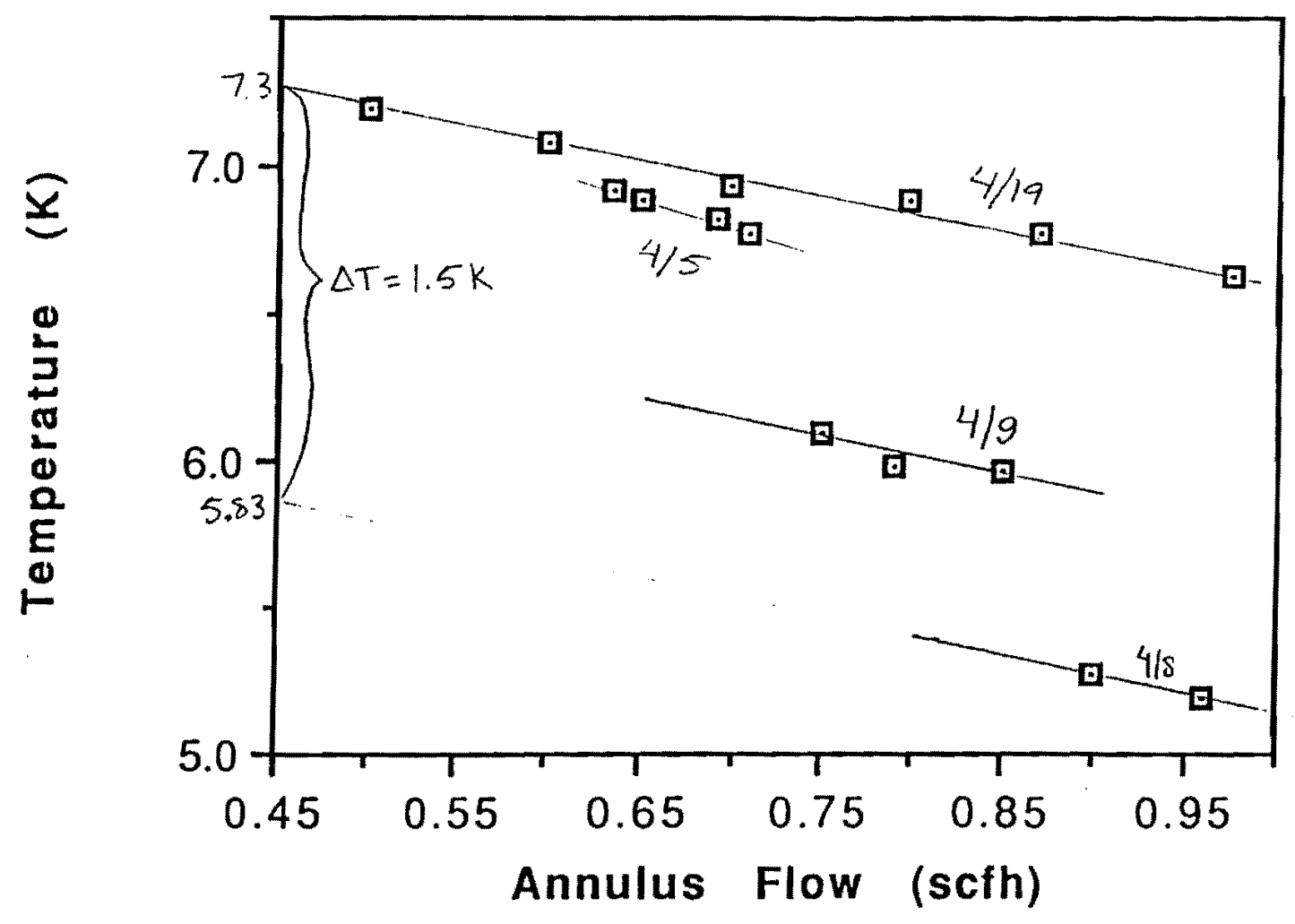




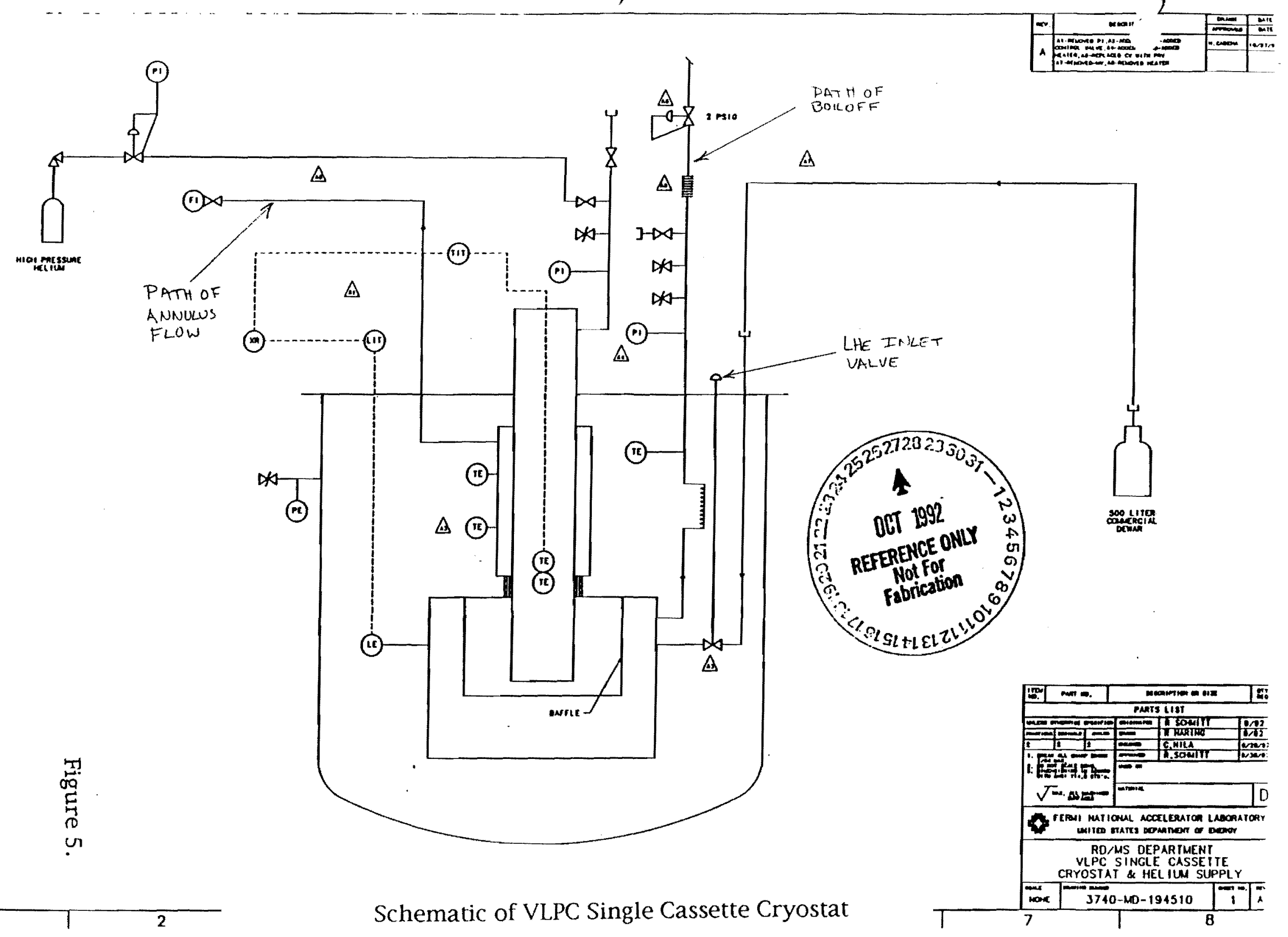




\begin{tabular}{|c|c|c|c|c|c|c|}
\hline $4 / 19 / 93$ & & & & & & \\
\hline flow $=.972$ & annulus & & Temp & Temp & Temp & $\mathrm{LHe}$ \\
\hline time & flow & tree res & Measured & Calculated & $\%$ Error & Level $(\%)$ \\
\hline $10: 10$ & 0.974 & 767.04 & 5.65 & 6.53 & -15.70 & 40.1 \\
\hline $10: 15$ & 0.972 & 666.02 & 5.91 & 6.54 & -10.70 & 38.6 \\
\hline $10: 21$ & 0.972 & 608.08 & 6.09 & 6.55 & -7.53 & 37.0 \\
\hline $10: 25$ & 0.972 & 575.08 & 6.21 & 6.57 & -5.78 & 33.8 \\
\hline $10: 27$ & 0.972 & 565.00 & 6.25 & 6.57 & -5.20 & 33.1 \\
\hline $10: 30$ & 0.972 & 550.59 & 6.31 & 6.57 & -4.17 & 34.1 \\
\hline $10: 33$ & 0.972 & 537.51 & 6.36 & 6.57 & -3.38 & 33.1 \\
\hline $10: 37$ & 0.972 & 519.56 & 6.44 & 6.59 & -2.40 & 30.0 \\
\hline $10: 41$ & 0.969 & 511.42 & 6.47 & 6.61 & -2.11 & 27.3 \\
\hline $10: 46$ & 0.969 & 500.96 & 6.52 & 6.64 & -1.76 & 22.5 \\
\hline $10: 48$ & 0.966 & 497.32 & 6.54 & 6.65 & -1.67 & 21.1 \\
\hline $10: 51$ & 0.974 & 486.61 & 6.59 & 6.61 & -0.37 & 25.4 \\
\hline $10: \overline{55}$ & 0.974 & 484.59 & 6.60 & 6.60 & -0.04 & 27.5 \\
\hline $10: 58$ & 0.974 & 483.79 & 6.60 & 6.59 & 0.14 & 29.0 \\
\hline $11: 10$ & 0.974 & 481.31 & 6.62 & 6.58 & 0.59 & 32.1 \\
\hline $11: 34$ & 0.972 & 480.41 & 6.62 & 6.62 & 0.04 & 25.1 \\
\hline $11: 38$ & 0.972 & 479.33 & 6.63 & 6.63 & -0.05 & 23.1 \\
\hline $11: 41$ & 0.974 & 479.21 & 6.63 & 6.64 & -0.19 & 20.9 \\
\hline $11: 48$ & 0.974 & 468.78 & 6.68 & 6.62 & 0.92 & 24.6 \\
\hline $11: 50$ & 0.870 & 461.34 & 6.72 & 6.75 & -0.46 & 22.7 \\
\hline $11: 54$ & 0.870 & 457.64 & 6.74 & 6.76 & -0.28 & 21.3 \\
\hline $11: 56$ & 0.870 & 456.25 & 6.75 & 6.77 & -0.38 & 18.7 \\
\hline $12: 00$ & 0.870 & 454.70 & 6.75 & 6.76 & -0.14 & 20.2 \\
\hline $12: 04$ & 0.870 & 455.52 & 6.75 & 6.75 & -0.06 & 21.9 \\
\hline $12: 06$ & 0.870 & 456.10 & 6.75 & 6.76 & -0.25 & 20.2 \\
\hline $12: 08$ & 0.870 & 455.99 & 6.75 & 6.76 & -0.22 & 20.4 \\
\hline $12: 10$ & 0.870 & 454.76 & 6.75 & 6.76 & -0.11 & 20.6 \\
\hline $12: 13$ & 0.870 & 454.92 & 6.75 & 6.76 & -0.05 & 21.5 \\
\hline$\langle 12: 14\rangle$ & 0.870 & 455.90 & 6.75 & 6.76 & -0.15 & 21.2 \\
\hline $12: 15$ & 0.870 & 453.90 & 6.76 & 6.76 & -0.01 & 21.0 \\
\hline $12: 17$ & 0.870 & 453.60 & 6.76 & 6.76 & -0.03 & 20.4 \\
\hline$\langle 12: 19\rangle$ & 0.870 & 455.40 & 6.75 & 6.76 & -0.21 & 20.0 \\
\hline $12: 22$ & 0.870 & 454.73 & 6.75 & 6.77 & -0.23 & 19.1 \\
\hline$\langle 12: 23\rangle$ & 0.870 & 452.60 & 6.77 & 6.78 & -0.15 & 18.0 \\
\hline $12: 25$ & 0.870 & 454.40 & 6.76 & 6.78 & -0.35 & 17.3 \\
\hline $12: 26$ & 0.870 & 451.30 & 6.77 & 6.78 & -0.11 & 17.2 \\
\hline $12: 28$ & 0.870 & 452.05 & 6.77 & 6.79 & -0.28 & 15.8 \\
\hline \multirow[t]{2}{*}{$12: 30$} & 0.870 & 450.80 & 6.78 & 6.79 & -0.23 & 15.2 \\
\hline & 0.870 & 453.20 & 6.76 & 6.81 & -0.70 & 11.8 \\
\hline $12: 45$ & 0.796 & 448.79 & 6.79 & 6.77 & 0.18 & 33.9 \\
\hline $12: 48$ & 0.796 & 445.23 & 6.81 & 6.77 & 0.57 & 35.1 \\
\hline $12: 51$ & 0.796 & 448.00 & 6.79 & 6.77 & 0.25 & 34.0 \\
\hline $12: 59$ & 0.796 & 446.06 & 6.80 & 6.76 & 0.61 & 36.4 \\
\hline
\end{tabular}


flow/LHeLev./temp.Data(4/19/93)

\begin{tabular}{|c|c|c|c|c|c|c|}
\hline $13: 03$ & 0.796 & 445.34 & 6.81 & 6.76 & 0.65 & 36.2 \\
\hline $13: 04$ & 0.796 & 445.80 & 6.80 & 6.77 & 0.43 & 33.9 \\
\hline $13: 09$ & 0.796 & 443.97 & 6.81 & 6.77 & 0.58 & 33.9 \\
\hline $13: 14$ & 0.796 & 444.65 & 6.81 & 6.79 & 0.30 & 31.2 \\
\hline $13: 17$ & 0.796 & 443.93 & 6.81 & 6.80 & 0.23 & 29.6 \\
\hline $13: 20$ & 0.796 & 443.85 & 6.81 & 6.80 & 0.19 & 29.0 \\
\hline $13: 25$ & 0.796 & 442.90 & 6.82 & 6.82 & -0.05 & 25.1 \\
\hline \multirow[t]{3}{*}{$13: 26$} & 0.796 & 442.25 & 6.82 & 6.83 & -0.06 & 24.2 \\
\hline & 0.796 & 443.90 & 6.81 & 6.83 & -0.31 & 22.9 \\
\hline & 0.796 & 441.40 & 6.83 & 6.84 & -0.13 & 22.5 \\
\hline \multirow[t]{3}{*}{$13: 27$} & 0.796 & 442.79 & 6.82 & 6.84 & -0.28 & 22.1 \\
\hline & 0.796 & 440.70 & 6.83 & 6.84 & -0.13 & 21.8 \\
\hline & 0.796 & 442.60 & 6.82 & 6.84 & -0.31 & 21.5 \\
\hline \multirow[t]{2}{*}{$13: 32$} & 0.796 & 440.77 & 6.83 & 6.85 & -0.26 & 20.2 \\
\hline & 0.796 & 442.10 & 6.82 & 6.86 & -0.48 & 18.9 \\
\hline $13: 33$ & 0.796 & 439.60 & 6.84 & 6.86 & -0.26 & 19.0 \\
\hline \multirow[t]{2}{*}{$13: 35$} & 0.796 & 439.65 & 6.84 & 6.87 & -0.39 & 17.4 \\
\hline & 0.796 & 440.70 & 6.83 & 6.87 & -0.62 & 15.7 \\
\hline \multirow[t]{2}{*}{$13: 37$} & 0.796 & 438.20 & 6.85 & 6.86 & -0.22 & 18.1 \\
\hline & 0.796 & 436.00 & 6.86 & 6.86 & 0.04 & 19.0 \\
\hline $13: 40$ & 0.796 & 430.00 & 6.89 & 6.85 & 0.70 & 20.9 \\
\hline $13: 41$ & 0.796 & 430.10 & 6.89 & 6.90 & -0.15 & 10.4 \\
\hline \multirow[t]{2}{*}{$13: 43$} & 0.796 & 430.86 & 6.89 & 6.85 & 0.57 & 20.2 \\
\hline & 0.796 & 431.00 & 6.89 & 6.85 & 0.56 & 20.2 \\
\hline $13: 45$ & 0.796 & 430.50 & 6.89 & 6.85 & 0.59 & 20.0 \\
\hline $13: 50$ & 0.796 & 441.20 & 6.83 & 6.76 & 1.01 & 36.4 \\
\hline $13: 55$ & 0.796 & 443.76 & 6.81 & 6.76 & 0.86 & 37.2 \\
\hline $13: 59$ & 0.796 & 442.76 & 6.82 & 6.77 & 0.81 & 35.6 \\
\hline $14: 03$ & 0.796 & 444.32 & 6.81 & 6.82 & -0.14 & 25.4 \\
\hline $14: 05$ & 0.796 & 443.85 & 6.81 & 6.77 & 0.62 & 34.3 \\
\hline $14: 07$ & 0.796 & 445.52 & 6.80 & 6.78 & 0.30 & 32.1 \\
\hline $14: 10$ & 0.796 & 444.31 & 6.8 .1 & 6.80 & 0.19 & 29.5 \\
\hline $14: 12$ & 0.796 & 443.94 & 6.81 & 6.80 & 0.18 & 29.0 \\
\hline $14: 15$ & 0.796 & 443.85 & 6.81 & 6.80 & 0.18 & 28.9 \\
\hline $14: 17$ & 0.796 & 443.34 & 6.82 & 6.81 & 0.09 & 27.2 \\
\hline $14: 20$ & 0.796 & 442.52 & 6.82 & 6.82 & -0.03 & 24.9 \\
\hline $14: 23$ & 0.796 & 442.44 & 6.82 & 6.83 & -0.18 & 22.9 \\
\hline \multirow[t]{2}{*}{$14: 25$} & 0.796 & 442.87 & 6.82 & 6.85 & -0.41 & 20.5 \\
\hline & 0.796 & 440.90 & 6.83 & 6.85 & -0.29 & 20.0 \\
\hline $14: 27$ & 0.796 & 441.47 & 6.83 & 6.85 & -0.35 & 19.9 \\
\hline $14: 30$ & 0.796 & 441.52 & 6.83 & 6.86 & -0.46 & 18.5 \\
\hline $14: 31$ & 0.796 & 440.79 & 6.83 & 6.87 & -0.55 & 16.6 \\
\hline $14: 33$ & 0.796 & 435.09 & 6.86 & 6.85 & 0.21 & 20.2 \\
\hline $14: 35$ & 0.796 & 431.89 & 6.88 & 6.84 & 0.60 & 21.7 \\
\hline $14: 37$ & 0.796 & 431.95 & 6.88 & 6.84 & 0.58 & 21.4 \\
\hline $14: 38$ & 0.796 & 432.45 & 6.88 & 6.85 & 0.50 & 21.0 \\
\hline
\end{tabular}




\begin{tabular}{|c|c|c|c|c|c|c|}
\hline $14: 39$ & 0.796 & 432.72 & 6.88 & 6.85 & 0.43 & 20.4 \\
\hline $14: 40$ & 0.796 & 432.58 & 6.88 & 6.85 & 0.43 & 20.2 \\
\hline $14: 42$ & 0.796 & 432.61 & 6.88 & 6.85 & 0.40 & 19.9 \\
\hline $14: 45$ & 0.699 & 425.02 & 6.92 & 6.98 & -0.88 & 16.2 \\
\hline $14: 48$ & 0.699 & 423.56 & 6.93 & 6.99 & -0.83 & 15.2 \\
\hline $14: 50$ & 0.699 & 421.49 & 6.95 & 6.99 & -0.67 & 14.9 \\
\hline $14: 59$ & 0.699 & 429.74 & 6.90 & 6.89 & 0.04 & 32.9 \\
\hline $15: 02$ & 0.699 & 427.20 & 6.91 & 6.90 & 0.10 & 30.9 \\
\hline $15: 04$ & 0.699 & 426.31 & 6.92 & 6.91 & 0.02 & 29.0 \\
\hline $15: \overline{06}$ & 0.699 & 425.37 & 6.92 & 6.92 & -0.02 & 27.4 \\
\hline $15: 09$ & 0.699 & 424.03 & 6.93 & 6.94 & -0.11 & 24.8 \\
\hline $15: 11$ & 0.699 & 423.41 & 6.93 & 6.95 & -0.30 & 21.7 \\
\hline $15: 13$ & 0.699 & 423.09 & 6.94 & 6.96 & -0.39 & 20.2 \\
\hline $15: 15$ & 0.699 & 422.23 & 6.94 & 6.96 & -0.32 & 20.2 \\
\hline $15: 19$ & 0.600 & 418.49 & 6.96 & 7.03 & -0.95 & 29.0 \\
\hline $15: 22$ & 0.600 & 418.92 & 6.96 & 6.97 & -0.12 & 40.0 \\
\hline $15: 25$ & 0.600 & 419.05 & 6.96 & 6.88 & 1.19 & 56.8 \\
\hline $15: 27$ & 0.600 & 433.75 & 6.87 & 6.89 & -0.28 & 54.2 \\
\hline $15: 30$ & 0.600 & 424.87 & 6.92 & 6.89 & 0.47 & 54.0 \\
\hline $15: 32$ & 0.600 & 426.79 & 6.91 & 6.91 & 0.08 & 51.2 \\
\hline $15: 35$ & 0.600 & 419.51 & 6.96 & 6.91 & 0.62 & 50.0 \\
\hline $15: 37$ & 0.600 & 419.10 & 6.96 & 6.92 & 0.57 & 49.0 \\
\hline $15: 42$ & 0.600 & 417.28 & 6.97 & 6.96 & 0.21 & 42.4 \\
\hline $15: 45$ & 0.600 & 412.52 & 7.00 & 6.96 & 0.55 & 41.4 \\
\hline $15: 49$ & 0.600 & 408.70 & 7.02 & 6.99 & 0.54 & 36.9 \\
\hline $15: 52$ & 0.600 & 407.45 & 7.03 & 7.00 & 0.40 & 33.6 \\
\hline $15: 54$ & 0.600 & 407.62 & 7.03 & 7.02 & 0.21 & 31.4 \\
\hline $15: 56$ & 0.600 & 403.80 & 7.06 & 7.03 & 0.37 & 29.0 \\
\hline $15: 58$ & 0.600 & 403.02 & 7.06 & 7.05 & 0.20 & 26.0 \\
\hline $16: 00$ & 0.600 & 402.44 & 7.06 & 7.06 & 0.07 & 23.6 \\
\hline $16: 02$ & 0.600 & 400.42 & 7.08 & 7.07 & 0.09 & 21.5 \\
\hline $16: 03$ & 0.600 & 400.81 & 7.08 & 7.08 & -0.04 & 20.2 \\
\hline $16: 05$ & 0.600 & 399.97 & 7.08 & 7.08 & -0.06 & 19.0 \\
\hline $16: 10$ & 0.499 & 398.74 & 7.09 & 7.16 & -0.99 & 27.0 \\
\hline $16: 14$ & 0.499 & 392.73 & 7.13 & 7.15 & -0.24 & 29.4 \\
\hline $16: 17$ & 0.499 & 388.44 & 7.16 & 7.22 & -0.92 & 15.3 \\
\hline $16: 19$ & 0.499 & 380.98 & 7.21 & 7.22 & -0.16 & 15.8 \\
\hline $16: 21$ & 0.499 & 380.07 & 7.21 & 7.23 & -0.24 & 13.7 \\
\hline $16: 22$ & 0.499 & 379.06 & 7.22 & 7.25 & -0.33 & 11.2 \\
\hline $16: 24$ & 0.499 & 384.32 & 7.19 & 7.19 & -0.03 & 21.8 \\
\hline $16: 26$ & 0.499 & 382.53 & 7.20 & 7.20 & 0.02 & 20.2 \\
\hline $16: 27$ & 0.499 & 381.31 & 7.21 & 7.21 & -0.04 & 17.8 \\
\hline $16: 29$ & 0.499 & 381.40 & 7.21 & 7.21 & -0.12 & 16.9 \\
\hline $16: 30$ & 0.499 & 381.24 & 7.21 & 7.21 & -0.09 & 17.1 \\
\hline
\end{tabular}

